\title{
Characterizing the Nano and Micro Structure of Concrete to Improve its Durability
}

\author{
P.J.M. Monteiro ${ }^{1 *}$, A.P. Kirchheim ${ }^{1}$, S. Chae ${ }^{1}$, P. Fischer ${ }^{2}$, A.A. MacDowell ${ }^{3}$, \\ E. Schaible ${ }^{3}$, H.R. Wenk ${ }^{4}$ \\ ${ }^{1}$ Department of Civil and Environmental Engineering, University of California at Berkeley \\ ${ }^{2}$ Center for X-ray Optics, Lawrence Berkeley National Laboratory, Berkeley, CA USA \\ ${ }^{3}$ Advanced Light Source, Lawrence Berkeley National Laboratory, USA \\ ${ }^{4}$ Earth and Planetary Science, University of California at Berkeley
}

\begin{abstract}
New and advanced methodologies have been developed to characterize the nano and microstructure of cement paste and concrete exposed to aggressive environments. High resolution full-field soft X-ray imaging in the water window is providing new insight on the nano scale of the cement hydration process, which leads to a nano-optimization of cement-based systems.. Hard X-ray microtomography images on ice inside cement paste and cracking caused by the alkali-silica reaction (ASR) enables three-dimensional structural identification. The potential of neutron diffraction to determine reactive aggregates by measuring their residual strains and preferred orientation is studied. Results of experiments using these tools will be shown on this paper.
\end{abstract}

Keywords: concrete, durability, microscopy, neutron, tomography, X-ray

\section{Introduction}

Classical studies of the microstructure of cement paste and concrete have been largely done using electron microscopy. Transmission electron microscopy has been used to investigate the complex nanostructure of calcium silicate hydrates, and scanning electron microscopy (both in secondary and backscattered mode) is frequently used to characterize the phase distributions and assess damage of the material exposed to aggressive environments. Both these techniques normally require using a high vacuum which can modify or even damage the fragile hydration products. The existence of high vacuum also prevents the in situ imaging of the hydration reactions. X-ray based imaging techniques, which are abundantly available at large scale synchrotron radiation facilities, have the potential to revolutionize the current understanding of hydration mechanisms of cements. Neutron scattering has been used to study the hydration of cement minerals and in the present paper shows how neutron diffraction can be employed to

\footnotetext{
* Corresponding author: Paulo J. M. Monteiro, Department of Civil Engineering, University of California, 721

Davis Hall, Berkeley, CA 94720.phone: +1 5106438251 e-mail: monteiro@ce.berkeley.edu
} 
measure the degree of deformation and reactivity in granitic rocks, a critical information in the study of alkali silica reaction.

Recent research using the full-field soft transmission X-ray microscope XM-1 located at beamline 6.1.2 at the Advanced Light Source (ALS) in Berkeley, California, and operated by the Center for X-ray Optics at Lawrence Berkeley National Laboratory since 1994, is described below. Originally designed for the observation of biological cells in their natural, wet state at atmospheric pressure, this microscope has wide application in nano-sciences, such as nanomagnetism and nano-materials sciences. Because this technique allows high-resolution soft Xray imaging of cement in aqueous solution and of the early formation of the hydration products, since 1998 numerous studies on cementitious materials have been carried out (see Ref. [1-11]).

Characterizing the three-dimensional network of microcracks using non-destructive techniques has proved challenging. The traditional method of first impregnating the concrete and then cutting and polishing the sample may introduce artifacts. In addition, all observations are limited to whatever can be determined from the surface of the sample; any three-dimensional analysis is impossible.

Microtomography has produced insightful information on the topology of cracks in the cement paste matrix and how they are deflected by the dispersed hard inclusions. This study reports on current research using the Hard X-ray Microtomography (XMT) located at beamline 8.3.2 at the ALS, which analyzes images obtained of cement paste exposed to freezing conditions and of three-dimensional cracks forming in mortar, as a result of the alkali-aggregate reaction.

Neutron scattering can provide unique information on potentially reactive aggregates for concrete. In many rocks, minerals exhibit a preferred orientation pattern, and this is expressed in anisotropy of physical properties. It is recognized that the deformation state, grain size, and development of foliation in granitic rocks used as aggregate in concrete influence the alkali-silica reaction. By performing an analysis of preferred orientation in conjunction with expansion tests, Monteiro et al. [12] showed that there is a quantitative relationship between the degree of deformation and reactivity in granitic rocks. In this paper, we summarize our on-going microstructural characterization of reactive rocks by neutron diffraction and transmission electron microscopy (TEM), observing dislocations and determining dislocation densities of quartz grains in granitic rocks of different deformation states.

\section{Soft X-ray Microscopy}

Soft X-ray microscopy uses advances in nanotechnology to provide high quality X-ray optical components. Fresnel zone plates, i.e., circular gratings with a radially increasing line density, are used to focus the X-rays, which constitute the basis for performing X-ray microscopy because with a refractive index close to unity, conventional lenses cannot be applied. State-of-the-art Fresnel zone plate optics have now achieved a $15 \mathrm{~nm}$-spatial resolution [13, 14]. The optical design for the soft X-ray microscope used here is an X-ray analogue to a conventional light microscope, whereby Fresnel zone plates are used for the condenser and objective lenses (see Figure 1).

Because the wavelength is dependent on its focal length (together with a pinhole close to the specimen as a monochromator with a spectral resolution $\mathrm{E} / \Delta \mathrm{E} \sim 500$ ), $\mathrm{X}$-rays emitted from a bending magnet source propagate to a condenser zone plate, which provides a partially incoherent hollow-cone illumination of the sample. The current condenser zone plate has an 
outer diameter of about $9 \mathrm{~mm}$, about 50,000 rings, and an outermost zone width $\Delta \mathrm{r}$ of about 40 $\mathrm{nm}$. The X-rays penetrating the specimen are projected onto an X-ray sensitive CCD by a micro zone plate, which is positioned at its focal length downstream of the sample; this creates a fullfield image. The backside-illuminated, thinned CCD has 2048x2048 pixels, with a physical pixel size of $13.5 \mu \mathrm{m}$. Because of the high flux of synchrotron radiation, typical exposure times per image are only a few seconds, covering a field of view of about $10 \mu \mathrm{m}$ diameter at a magnification of about 2500 . The micro zone plate, i.e., the high resolution objective lens, has a diameter of about 30-60 $\mu \mathrm{m}$, typically about 500 rings, and an outermost zone width $\Delta \mathrm{r}$ down to $15 \mathrm{~nm}$, a critical factor in determining the spatial resolution possible using soft X-ray microscopy.

Figure 2 shows the main steps necessary in preparing the samples to be subjected to soft x-ray microscopy. The samples must be highly diluted for transmission of the soft X-rays. In order to retard the dissolution of the cement minerals in the highly diluted samples, and to provide calcium and sulfate ions for the reactions, a solution saturated with $\mathrm{Ca}(\mathrm{OH})_{2}$ and $\mathrm{CaSO}_{4} \cdot 2 \mathrm{H}_{2} \mathrm{O}$ was used as the liquid media $(\mathrm{pH}=12.3)$. The solution was prepared using freshly boiled deionized water inside a glove bag filled with nitrogen gas to avoid carbonation. Teflon and polyethylene flasks were used to prepare the solution to avoid alkali-silica reaction. The solid particles were mixed in the solution for $60 \mathrm{~s}$ inside a test tube, and approximately $2 \mathrm{ml}$ of the mixture was centrifuged for $15 \mathrm{~s}$. A small droplet — around $2 \mu \mathrm{l}$ - was taken from the supernatant with a micropipette and squeezed between two silicon nitride windows in a metallic sample holder, which was then placed in the microscope for examination.

\section{Images and results}

Shrinkage of concrete on drying frequently leads to cracking. Recognized as a serious problem in concrete design and construction practice, especially in regard to pavements, floors, and relatively thin structural members, one solution is to use expansive cements (e.g., Type $\mathrm{K}$ cement, which contains $\mathrm{C}_{4} \mathrm{~A}_{3} \overline{\mathrm{S}}$ as the principal source of the reactive aluminate) or expansive admixtures [15]. Expansive cements and admixtures, unlike Portland cement, expand during the early hydration period after setting. The expansion is a consequence of the formation of ettringite (calcium sulfoaluminate hydrate) in considerable quantities during the first few days of curing. Two separate research projects using soft $\mathrm{x}$-ray microscopy to study this phenomenon are reported therein and preliminary results presented below.

In the first series of tests, the hydration of the $\mathrm{C}_{4} \mathrm{~A}_{3} \overline{\mathrm{S}}$ in a $\mathrm{Ca}(\mathrm{OH})_{2}+\mathrm{CaSO}_{4} \cdot 2 \mathrm{H}_{2} \mathrm{O}$ saturated solution was analyzed. The solution/solid materials ratio was $50 \mathrm{ml} / \mathrm{g}$. The morphology of this compound in absence and in presence of $10 \%$ of $\mathrm{Ca}(\mathrm{OH})_{2}$ is presented over the time. Figures $3 \mathrm{a}$ and $3 \mathrm{~b}$ show a sequence of large ribbons arranged in a fan-like configuration or cross shape for a saturated $\mathrm{Ca}(\mathrm{OH})_{2}$ solution, while in the presence of $10 \% \mathrm{Ca}(\mathrm{OH})_{2}$ the same ribbons are formed, but at a smaller size; this is assumed to be a retardation of the $\mathrm{C}_{4} \mathrm{~A}_{3} \overline{\mathrm{S}}$ hydration. The same retardation was observed by Mehta [16].

In the second series of tests, one type of expansive admixtures based on calcium sulfoaluminate was blended with Type I-II cement to analyze how expansive admixture works alone and in combination with Portland cement. The expansive admixture is used to reduce shrinkage and the associated cracking, it is a suitable product for multiple applications, including concrete pavement. Figure 4 shows soft X-ray images of Type I-II cement (Figure 4a) and pure expansive 
agent (Fig. 4b) after 35 minutes in solution. Figures 4c and d indicate how the expansive admixture modifies the microstructure. The solution/solid materials ratio was $10 \mathrm{ml} / \mathrm{g}$.

The reaction between calcium, sulfate, aluminate, and hydroxyl ions within a few minutes of cement hydration resulted in needle-shaped crystals of a calcium sulfoaluminate hydrate (ettringite) appearing before 21 minutes (see Fig. 4c). When cement is blended with $6 \%$ of expansive admixture and hydrated, basically ettringite (needles) and calcium hydroxide (platelets) were formed close to the surface of the cement particles, which agree with Nagataki and Gomi [17]. Differently those which can be seen in Fig. 4a, the platelets are formed close to the surface of the particle, this is important in order to achieve the desired amount of expansion.

\section{X-ray Microtomography}

In synchrotron XMT, a wide parallel X-ray beam passes through the sample, and the transmitted fraction is detected as an image by a charge couple device (CCD) area detector. The sample is mounted on a stage with four degrees of freedom: $x, y$, and $z$ transition for positioning and centering of the sample, and rotation for data acquisition. The rotary stage allows for many X-ray transmission images of the sample to be recorded for a series of orientations. According to the Nyquist theorem, the minimum number of radiographs needed for a full reconstruction within 180 degrees of rotation is $N \pi / 2$, where $N$ is width of the sample in pixels. In practice, little difference is observed for image numbers $>\mathrm{N} \pi / 4$.

At the ALS superbend beamline 8.3.2, the transmitted beam is detected by a scintillator that converts X-rays to visible light, which is imaged by magnifying lenses onto a CCD. The available optics allow for spatial resolutions between $0.44 \mu \mathrm{m} / \mathrm{pixel}$ and $11.5 \mu \mathrm{m} / \mathrm{pixel}$. The monochromator is tunable to X-ray energies between $5 \mathrm{keV}$ and $35 \mathrm{keV}$ by means of a double multilayer mirror arrangement. The monochromator optics can also be removed from the beam, allowing a white light mode of operation that is broadly polychromatic in the $30-70 \mathrm{keV}$ range by means of metal filters.

Tomography imaging can be done either in absorption or phase contrast mode. In absorption mode, each pixel of the projection image represents the intensity of the transmitted beam that has not been absorbed by the sample. According to Beer's law :

$$
I(x)=I_{o} e^{-\mu x}
$$

Where $I(x)$ is the final intensity of the beam after the beam passes through sample thickness $x$, $I_{o}$ is the initial intensity of the beam, and $\mu$ is the material's linear attenuation coefficient, which can be expressed as:

$\mu=\sum f_{i} \mu_{i} \rho$

where $i$ represents each atomic element, $f_{i}$ is the atomic weight fraction of the element in the material, $\mu_{i}$ is the mass attenuation coefficient at the beam energy, and $\rho$ is the density of the material.

The attenuated value for each pixel, $P\left(\varphi, x^{\prime}\right)$ is then determined by:

$$
P\left(\varphi, x^{\prime}\right)=-\ln \left[I\left(\varphi, x^{\prime}\right) / I_{o}\left(\varphi, x^{\prime}\right)\right] \text {, }
$$


where for each angle of rotation for the sample, $\varphi, x^{\prime}$ indicates the axis parallel to the X-ray direction and perpendicular to the sample rotation; $I_{o}\left(\varphi, x^{\prime}\right)$ is the initial intensity of the beam, and $I\left(\varphi, x^{\prime}\right)$ is the intensity of the transmitted beam.

The attenuation can be expressed as an integral of the linear attenuation coefficient over the sample:

$P\left(\varphi, x^{\prime}, z\right)=\iint_{\infty} \mu(x, y, z) \delta\left(x \cos \varphi+y \sin \varphi-x^{\prime}\right) d x d y$

where the $\delta$ function defines the path of the X-rays through the sample, and $\mu$ is the material's linear attenuation coefficient. The function $P\left(\varphi, x^{\prime}, z\right)$ is known as the Radon transform or sinogram. Reconstruction involves inverting this equation to yield $\mu(x, y, z)$ at each voxel location [18]. Ultimately, the reconstruction procedure combines the two-dimensional projection images to yield a three-dimensional data set of the absorbance of the sample at each voxel. The processed data contains a series of cross-sectional slices (tomograms) at set height $z$ of the sample.

The absorption mode scanning is usually applied using monochromatic light. If polychromatic beam is used, Beer's law no longer applies because the high-energy X-rays penetrate farther than those of low energy. This causes the "beam hardening effect," making the resulting image less quantitative. Mathematical approximations are necessary to adjust for the beam hardening effect, and a rigorous reconstruction can only be made if it is assumed that all components of the sample have the same energy dependence of absorption.

The phase contrast mode relies on the variation of the real part of the index of refraction to provide image contrast. Materials differing in their refractive index result in differing phase shifts to the transmitted X-rays. The effect of the differing phase shifts is only seen at the edges between materials of different indices. The interaction of X-rays with differing phase shifts result in intensity cancellation or enhancement at the detector plane. Edges are seen as oscillating dark and bright lines and generally have much higher contrast than the corresponding absorption images. At X-ray wavelengths, the index of refraction is about 10-100 times larger than the absorption index. Thus the phase contrast mode of operation may, in principle, be more sensitive than the absorption mode. Phase contrast tomography is harder to interpret quantitatively, but progress is being made in this new area [19]. The phase contrast mode is useful for imaging light materials for which the absorption contrast is very small, such as for low density materials or for materials with very similar absorption properties but where small changes in the refraction index can be observed.

With the radiograph size limit of 4000 pixels by 3000 pixels, and the source beam size being less than $4 \mathrm{~cm}$ wide and usually several millimeters high, the choice of magnification becomes a tradeoff between the desired magnification and the scale of the sample to be tested. The sample must fit completely within the width of the produced radiograph, otherwise the tomographic reconstruction becomes more approximate. Although smaller samples may allow for greater magnification and finer dimensions of the smallest observable detail, the data might not be appropriate given that concrete is generally used in the construction of large structures. Using larger samples, however, results in lower magnification and coarser dimensions of the smallest observable particle size. 
As an example of the application of microtomography to study the durability of concrete, reported herein are preliminary conclusions of the analyses of images obtained in the study of in situ ice formation in entrained air-voids in hydrated cement paste. For this experiment, XMT scans were performed on air-entrained, hydrated cement paste cast in $0.25 \mathrm{~mm}$ outer diameter glass capillary tube while subjecting the sample to several cycles of evaporated liquid nitrogen gas cooling and ambient thawing. The samples were frozen using an Oxford 600-series Cryostream. This unit is designed to cool small crystals for protein crystallography by the production of a jet of cold nitrogen gas with a coaxial outer sheath of dry nitrogen for frost prevention. The scan settings were optimized for both absorption and phase contrast imaging. The absorption contrast provided imaging of the dense hydrated cement matrix, while the phase contrast provided imaging of the ice-air phases that would coexist in the partially filled air void. A $13 \mathrm{KeV}$ monochromatic $\mathrm{x}$-ray energy provided sufficient penetration through the hydrated cement paste, while an approximately $30 \mathrm{~cm}$ sample-to-detector distance enhanced the phase contrast imaging and made visible the ice-air interface within the air voids. The magnification was set at 5.4x with the resulting pixel size of $1.54 \mu \mathrm{m} /$ pixel.

Figure 5 shows a cross section through one of the cement air voids. The donut shape is the cement material constrained within the glass tube. Figure 5a shows the sample when frozen, with dendritic ice growth obvious within the void. The sample was then allowed to warm up to room temperature (Figure 5b). The ice formation disappears and then reappears with a different structure as the sample was frozen once again (see Figure 5c). Figure 6 shows a threedimensional reconstruction of the air/ice interface in a single air void; it is roughly that of a spherical shell. Upon thawing, the interface vanished, confirming that the image of the ice was successful.

Microtomography is also useful for performing time-scale experiments. Here, a modified ASTM C-1260 test of aggregate reactivity was conducted. In this experiment, three 1 in. $x 1$ in. $x$ 12 in. mortar bars were cast following the specifications detailed in ASTM C-1260, using known inert aggregate, while $50 \%$ of the aggregates by weight were substituted with partially strained quartz sand. The goal was to supplement the two-week long gross numerical test method with visual representation of damage in progress. Considering the scale and the density of the sample, the samples were scanned under a white beam filtered with aluminum plates and under low magnification. The resulting spatial resolution was $11.55 \mu \mathrm{m} /$ pixel. The scans were obtained under phase contrast mode in an attempt to enhance crack widths smaller than spatial resolution. Because the 1 in. $x 1$ in. cross section of the mortar bars was wider than the beam width, a narrower sample (0.75 in. $x 0.75$ in. $x 9$ in.) was cast. The large bars and the smaller bar were subject to the same test conditions prescribed by ASTM C-1260. The larger bars were designed to provide strain data while the smaller bar would be used for imaging purposes. The smaller bar underwent a slightly amplified but comparable rate of expansion and was deemed to be a suitable representation of the standard-sized mortar bar expansion. Figure 7 shows internal sections of the same sample after 7 and 14 days. Close examination of the older sample shows the development of micro-cracks.

\section{Neutron Diffraction and Texture Analysis of Reactive Rocks}

The previous discussion has mainly focused on the application of synchrotron X-rays in cement research. Other opportunities are provided by neutron scattering (e.g. Wenk, [20]). Compared to $\mathrm{X}$-rays neutrons have low absorption for most elements and are thus ideal to investigate bulk 
samples with penetration of centimeters to tens of centimeters. It is easy to perform in situ experiments at low/high temperature and pressure and record changes during chemical reactions and thus to study kinetics. For cement the interaction of neutrons with hydrogen is of particular importance: Contrary to X-rays which scatter on electrons and thus hydrogen with one electron is largely invisible, neutrons scatter on the nucleus and scattering on hydrogen is of similar magnitude as for heavy elements. For hydrogen ( $\mathrm{H}$ with one proton) there is a large interaction and energy exchange with thermal neutrons resulting in strong inelastic scattering. For deuterium (D with a proton and a neutron) there is little inelastic interaction and compounds with D are preferred for crystallographic studies. Finally, for neutrons, scattering on the small nucleus the scattering factor does not decrease appreciably with scattering angle $2 \theta$ and thus high intensities are observed to small d-spacings, resulting in good resolution for structure refinements. The application of neutron scattering to cement has been reviewed by Livinston et al. [21].

Neutrons have been critical to determine the detailed crystal structures of ettringite [22] and portlandite [23] and were used for quantitative phase analysis of clinkers (e.g. Peterson et al. [24], Pritula [25]). Small angle scattering lends itself to quantify porosity in concrete (e.g. Allen et al. [26], Jennings et al. [27]) as well as to investigate the still enigmatic structure of C-S-H [see Ref. 28-31] and kinetic studies of hydration [32]. High resolution neutron strain diffractometers have been used to determine internal stresses imposed during freezing of concrete [33] and recently there has been interest in determining the nature of water bonds with inelastic neutron scattering [34], which is likely to become an important application with new high resolution instruments such as the Fourier Difference Specrometer (FDS) at LANSCE or similar instruments in ISIS [35]. Finally neutrons are very complementary to X-rays in tomography [36]. X-rays are excellent to determine high atomic number contrast such as steel reinforcements in concrete, or pore space. With neutrons it is possible to obtain 3D images of water migration and reactions of hydrous phases.

We have been making use of the time-of-flight neutron diffractometer HIPPO (High Pressure Preferred Orientation [37] for a rather unique concrete application. This instrument has a large sample chamber for ancillary equipment such as pressure cells and furnaces and many detectors for fast data acquisition (Fig. 8). It has been used to investigate structure changes in portlandite with pressure and temperature [38]. We used the capability that each detector records differently oriented crystals in the sample and crystal orientation patterns are related to the deformation history of a rock.

We have observed that the deformation state of a granitic rock has a profound impact on the long-term stability of concrete if used as aggregate due to enhanced susceptibility to the alkalisilica reaction $[39,40]$. An investigation of the microstructure of granitic rocks revealed that, as these rocks become progressively deformed from granite to mylonite and phyllonite they develop a microstructure that makes them increasingly unsuitable for use as aggregates. ASTM C 1260 expansion tests were performed and indicate expansion of 0.25 in granite up to 0.55 in phyllonite after 30 days. To obtain a bulk measure of the deformation state we have determined preferred orientation of mica with HIPPO. Mica platelets align during ductile deformation and this "directionality" is most commonly represented in pole figures. A pole figure is constructed by placing a polycrystalline sample inside a sphere and intersecting a crystal direction (e.g., the pole to the (001) platelet plane of mica) with the surface of the sphere. Pole densities can then be contoured and expressed as a smooth distribution, usually normalized such that contours express pole densities in multiples of a uniform distribution. These pole figures (Figure 9) document that 
preferred orientation increases from maximum pole densities of 1.4 m.r.d. in weakly deformed granite to 12 m.r.d. in phyllonite. Biotite preferred orientation (and thus overall deformation) correlates perfectly with the observed expansion (Fig. 10).

\section{Conclusions}

A well-documented understanding underlying cement hydration has remained elusive given the complexity of the reaction. The recent application of advanced methodologies has been developed to characterize the nano and microstructure of cement paste and concrete exposed to aggressive environments. High resolution full-field soft X-ray imaging in the water window is providing new insight into the cement hydration process on the nano scale. Preliminary results of studies using X-ray imaging to quantify this mechanism affords some clear benefits for the characterization of cement-based materials:

- Rapid data acquisition

- High (nanoscale) resolution

- Non-destructive examination

- Characterization of hydrated samples at normal temperature and pressure

Texture analysis of quartz grains showed no simple relationship between quartz preferred orientation and the susceptibility to the alkali-silica reaction. However, texture analysis of biotite, a common accessory mineral in granites, shows a linear relationship with reactivity. These results suggest that preferred orientation of biotite may be used to estimate the susceptibility of rocks containing both biotite and quartz to the alkali-silica reaction.

\section{Acknowledgment}

Paulo Monteiro and Rudy Wenk acknowledge the financial support from the National Science Foundation Grant 062464 and from KAUST. Ana Paula Kirchheim acknowledge the financial support of CAPES (Fundação Coordenação de Aperfeiçoamento de Pessoal de Nível Superior Ministério da Educação - Brasil). The soft X-ray microscope operation was supported by the U. S. Department of Energy, Office of Science, Basic Energy Sciences, Division of Materials Sciences and Engineering. The Advanced Light Source is supported by the Director, Office of Science, Office of Basic Energy Sciences, of the U.S. Department of Energy under Contract No. DE-AC02-05CH11231. The authors would like to thank Cruz Carlos and Roula Rbeiz for their assistance in the ASTM-1260 preparation and testing.

\section{References}

1. Kurtis, K. E., Monteiro, P. J. M., Brown, J. T., and Meyer-Ilse, W. Imaging of ASR gel by soft X-ray microscopy. Cement and Concrete Research, 1998. 28:411-421.

2. Kurtis, K. E., Monteiro, P. J. M., and Meyer-Ilse, W. Soft X-ray spectromicroscopy for in situ study of corrosion, Journal of Corrosion Science, 2000. 42(8):1327-1336.

3. Gartner, E. Kurtis, K. E., and Monteiro, P. J. M.. Proposed mechanism of C-S-H growth tested by X-ray microscopy, Cement and Concrete Research, 2000. 30(5):817-822.

4. Lamour, V. H. R., Monteiro, P. J. M., Scrivener, K. L., and Fryda, H. Microscopic studies of the early hydration of calcium aluminate cements. In: International Conference on Calcium Aluminate cement (CAC), July, 2001, Edinburgh. UK. Proceedings. p. 169-180. 
5. Kurtis, K. E., Collins, C. L., and Monteiro, P. J. M. The surface chemistry of the alkali-silica reaction: A critical evaluation and X-ray microscopy, Concrete Science and Engineering, 2002. 4:1-11.

6. Kurtis, K. E., and Monteiro, P. J. M.. Chemical additives to control expansion of alkali-silica reaction gel: proposed mechanisms of control, Journal of Materials Science, 2003. 38(9):2027-2036.

7. Juenger, M. C. G., Lamour, V., and Monteiro, P. J. M., Gartner, E. M., and Denbeaux, G. P. Direct observation of cement hydration by X-ray microscopy, Journal of Materials Science Letters, 2003. 22(19):1335-1337.

8. Juenger, M. C. G., Monteiro, P. J. M., Gartner, E. M., and Denbeaux, G. P.. A soft X-ray microscope investigation into the effects of calcium chloride on tricalcium silicate hydration, Cement and Concrete Research, 2005. 35(1):19-25.

9. Silva, D. A., and Monteiro, P. J. M. Hydration evolution of C3S - EVA composites analyzed by soft X-ray microscopy, Cement and Concrete Research, 2005. 35(2):351-357.

10. Silva, D. A., and Monteiro, P. J. M. The influence of polymers on the hydration of portland cement phases analyzed by soft X-ray transmission microscopy, Cement and Concrete Research, 2006. 36(8):1501-1507.

11. Silva, D. A. and Monteiro, P. J. M. Early formation of ettringite in tricalcium aluminate calcium hydroxide - gypsum dispersions, Journal of Ceramics Society, 2007. 90(2):614617.

12. Monteiro, P. J. M., Shomglin, K., Wenk, H.-R., and Hasparyk, N. P. Effect of Aggregate Deformation on Alkali-Silica Reaction. ACI Materials Journal, Farmington Hills Michigan, 2001. v. 98, n. 2, p. 179-183

13. Chao, W., Harteneck, B. H., Liddle, J. A., Anderson, E. H., and Attwood, D. T.. Soft x-ray microscopy at a spatial resolution better than $15 \mathrm{~nm}$, Nature, 2005. 435:1210-1213

14. Kim D.-H., Fischer, P., Chao, W., Anderson, E., Im, M.-Y., Shin, S.-C., and Choe, S.-B. Magnetic soft X-ray microscopy at $15 \mathrm{~nm}$ resolution probing nanoscale local magnetic hysteresis (invited), also selected in Virtual Journal of Nanoscale Science \& Technology, 2006. 13(17), May 1.

15. Mehta, P. K., and Monteiro, P. J. M. Microstructure, Properties and Materials, McGrawHill, Third Edition, 659 pp., 2006

16. Mehta, P.K., Effect of Lime on Hydration of Pastes Containing Gypsum and Calcium Aluminates or Calcium Sulfoaluminate. Journal of The American Ceramic Society, 1973. 56(6).

17. Nagataki, S. and H. Gomi, Expansive admixtures (mainly ettringite). Cement and Concrete Composites, 1998. 20(2-3): p. 163-170.

18. Kak. A. C., and Slaney, M. . Principles of Computerized Tomographic Imaging, Classics in Applied Mathematics; 2001. Vol. 33, Society for Industrial and Applied Mathematics, Philadelphia, PA.

19. Groso, A., Abela, R., and Stampanoni, M. Implementation of a fast method for high resolution phase contrast tomography, Optics Express, 2006. 14:8103-8110.

20. Wenk HR Edit. Neutron Scattering in Earth Sciences. Vol. 63 of Rev. Mineralogy and Geochemistry. Mineral. Soc. Amer. 471pp, 2006.

21. Livingston RA, Neumann DA, Allen AJ, Fitzgerald SA and Berliner R. Application of neutron scattering to Portland cement. Neutron News 11, 2000. 
22. Hartman, M. R. and Berliner, R. Investigation of the structure of ettringite by time-of-flight neutron powder diffraction. Cement and Concrete Research, 2006. 36, 364-370

23. Leinenweber K, Partin D. E., Schuelke U., O'Keeffe M, Von Dreele R.The structure of high pressure $\mathrm{Ca}(\mathrm{OD}) 2 \mathrm{II}$ from powder neutron diffraction: Relationship to the $\mathrm{ZrO} 2$ and EuI2 structures. J. Solid State Chem.1997. 132, 267-273

24. Peterson V, Hunter B, Ray A, Aldrige L., P. Rietveld refinement of neutron, synchrotron and combined powder diffraction data of cement clinker. Applied Phys. A, 2004, 74, s14091411.

25. Pritula, O. X-ray and neutron Rietveld quantitative phase analysis of industrial Portland cement clinkers. Powder Diffraction, 2004. 19(3)

26. Allen A. J., Windsor, C. G., Rainey V., Pearson D., Double D. D., Alford N. McN. A smallangle neutron-scattering study of cement porosities. J. Phys. D. 1982. 15, 1817-1833.

27. Jennings H.M., J.J. Thomas, J.S. Gevrenov, G. Constantinides, and F.-J. Ulm A multitechnique investigation of the nanoporosity of cement paste Cement and Concrete Research, 2007. 37, 329-336.

28. Allen A.J., Thomas J.J. and Jennings H.M. Composition and density of nanoscale calciumsilicate-hydrate in cement. Nature Materials, 2007. V. 6 April

29. Allen A.J and Thomas J.J. Analysis of cement paste and C-S-H gel by small-angle neutron scattering Cement and Concrete Research, 2007. 37, 319-324

30. Thomas J.J., Jennings H.M., Allen A.J. The surface area of cement paste as measured by neutron scattering - Evidence for two C-S-H morphologies. Cement and Concrete Research, 1998. 28 [6], 897-905

31. Thomas J.J., FitzGerald, S.A. Neumann, D. A. Livingston, R.A. The State of Water in Hydrating Tricalcium Silicate and Portland Cement Pastes as Measured by Quasi-Elastic Neutron Scattering Journal of the American Ceramic Society, 2001. 84 1811-16.

32. Fitzgerald S.A., Thomas, J.J. Neumann, D.A. Livingston R.A. A neutron scattering study of the role of diffusion in cement hydration Cement and Concrete Research, 2002. 32, 409413.

33. Schulson, E.M., Swainson, I.P., Holden, T.M. Internal stress within hardened cement paste induced through thermal mismatch: Calcium hydroxide versus calcium silicate hydrate. Cement and Concrete Research, 2001. 31, 1785-1791.

34. Padureanu, I., Aranghel, D., Rotarescu G, Dragolici, F., Turcanu, C., Kozlov, Z.A., Semonov, V.A. An inelastic neutron scattering study of the water dynamics in cement paste. Rom. J. Phys. 2005. 50, 561-574.

35. Parker, S.F. Inelastic Neutron Scattering Spectroscopy. In "Handbook of Vibrational Spectroscopy". Wiley. 2006

36. Lehmann E.H., Frei G.,. Kuehne, G. Boillat, P. The micro-setup for neutron imaging: A major step forward to improve the spatial resolution NUCLEAR INSTRUMENTS \& METHODS A, 2007. 576 389-396

37. Wenk H.-R., Lutterotti, L. Vogel, S. Texture analysis with the new HIPPO TOF diffractometer. Nucl. Instr. Methods A, 2003. 515, 575-588.

38. Xu H., Zhao, Y., Zhang, J., Hickmott, D.D., Daemen, L.L. In situ neutron diffraction study of deuterated portlandite $\mathrm{Ca}(\mathrm{OD}) 2$ at high pressure and temperature. Phys. Chem. Mineral. 2007. 34, 223-232. 
39. Monteiro P.J.M., K. Shomglin, H.-R. Wenk and N.P. Hasparyk. Effect of aggregate deformation on alkali-silica reaction. Am. Concr. Institute Materials Journal, 2001. 98, $179-183$

40. Wenk H.-R., Monteiro, P. Shomglin, K. Relationship between aggregate microstructure and concrete expansion. A case study of deformed granitic rocks from the Santa Rosa Mylonite Zone. J. Materials Science, 2007. 43:1278-1285

\section{Figures and Captions}

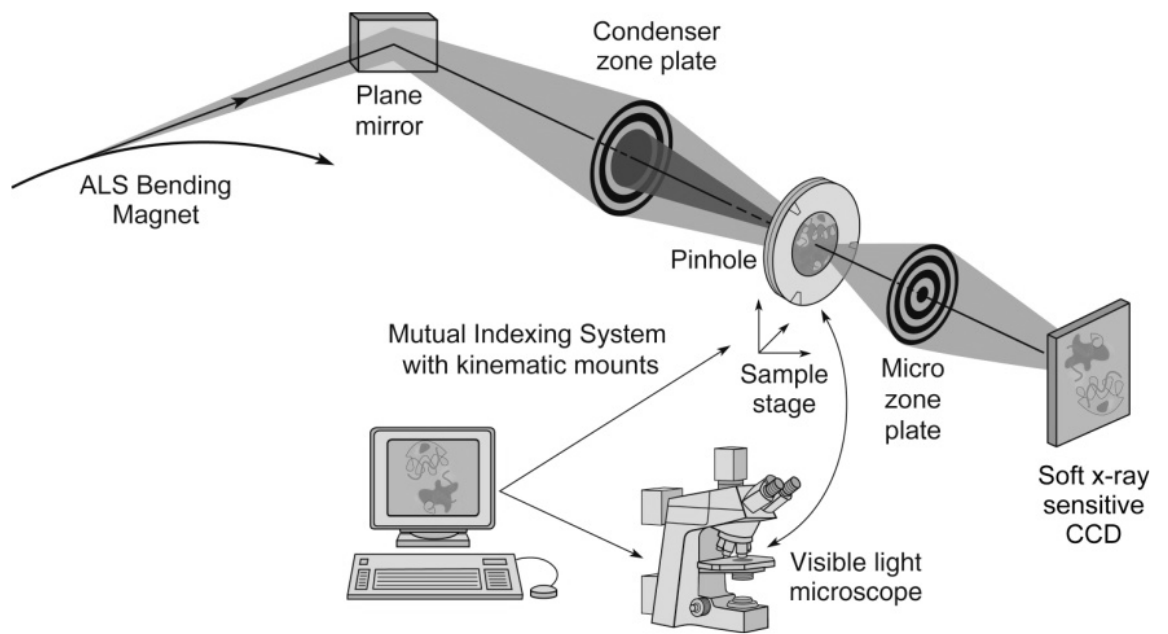

Figure 1 - Schematic layout of the soft X-ray microscopy beamline XM-1 at the ALS using $\mathrm{X}$-rays emitted from a bending magnet. The micro zone plate projects a full field image onto a CCD camera that is sensitive to soft X-rays. 


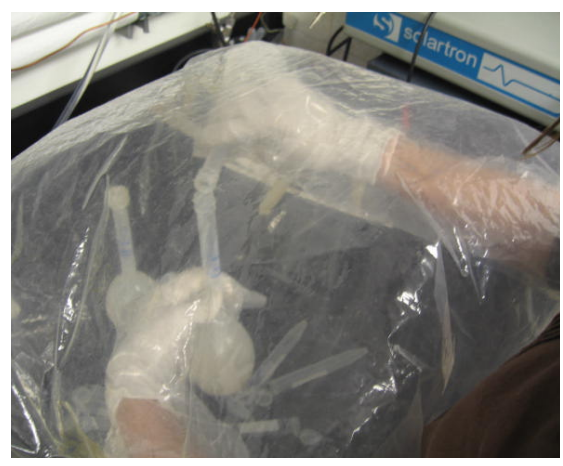

(a) preparation of the solutions

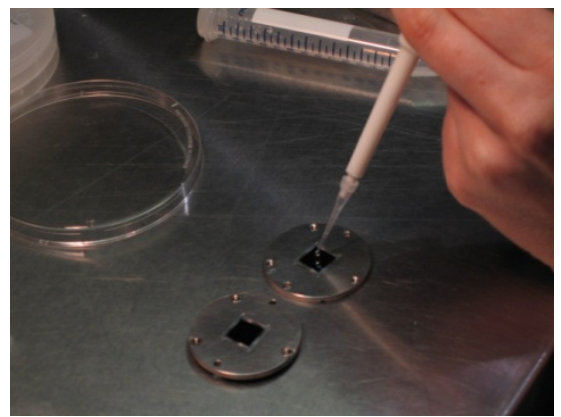

(c) placement of the cement mixture into the windows

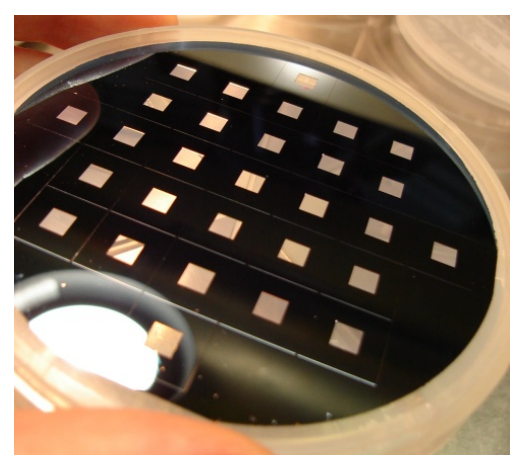

(b) silicon nitride windows

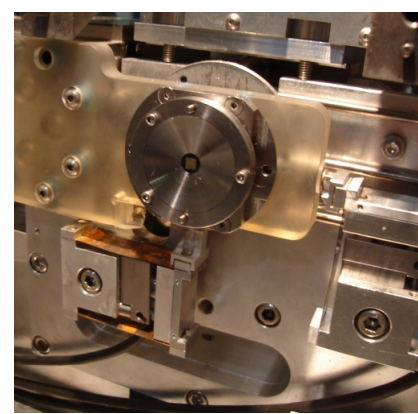

(e) sample holder in the soft $x$ ray microscope

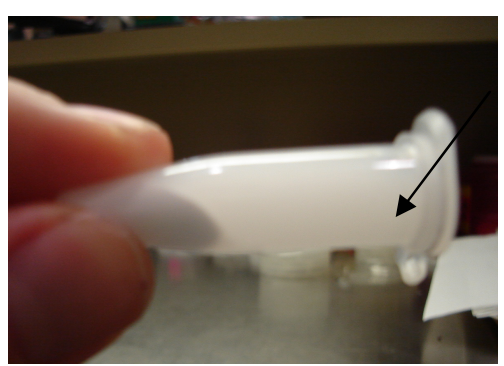

(c) aspect of the supernatant solution after centrifugation

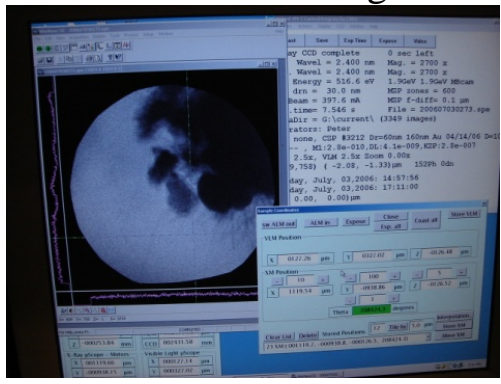

(f) data acquisition

Figure 2 - Sequence of sample preparation 


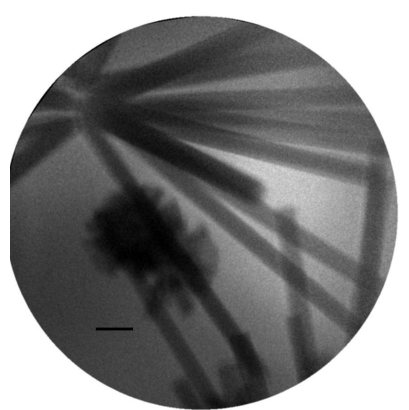

(a) $22 \mathrm{~min}$

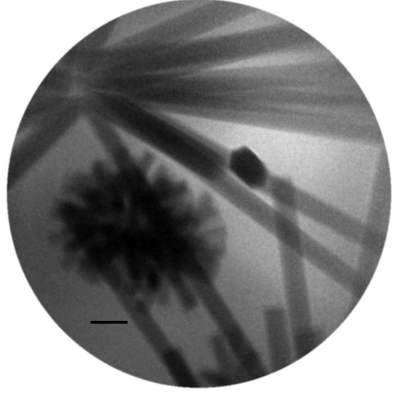

(b) $2 \mathrm{~h} 45 \mathrm{~min}$

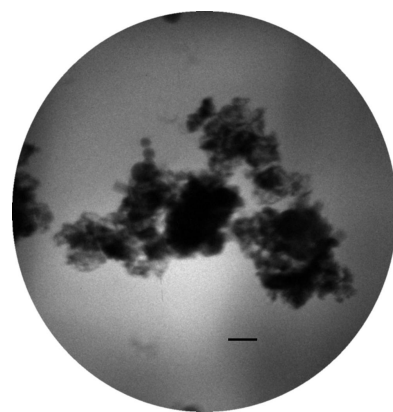

(c) $19 \min$

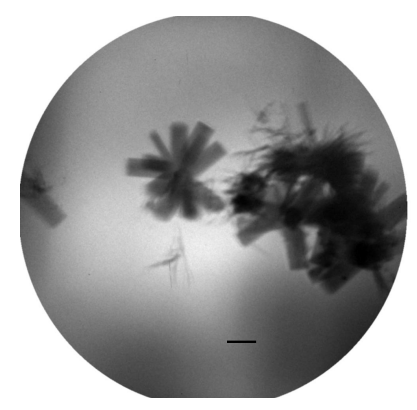

(d) $2 \mathrm{~h} 47 \mathrm{~min}$

Figure 3 - In situ soft x-ray images of hydrating $\mathrm{C}_{4} \mathrm{~A}_{3} \overline{\mathrm{S}}$ particles in a saturated calcium hydroxidecalcium sulfate solution, s/c= $50 \mathrm{ml} / \mathrm{g}$. Figs (a) and (b): hydration in $\mathrm{Ca}(\mathrm{OH})_{2}+\mathrm{CaSO}_{4} \cdot 2 \mathrm{H}_{2} \mathrm{O}$ saturated solution. Figs. (c) and (d): hydration in solutions with the presence of $10 \% \mathrm{Ca}(\mathrm{OH})_{2}$; Hydration time is indicated. Scale bar corresponds to $1 \mu \mathrm{m}$

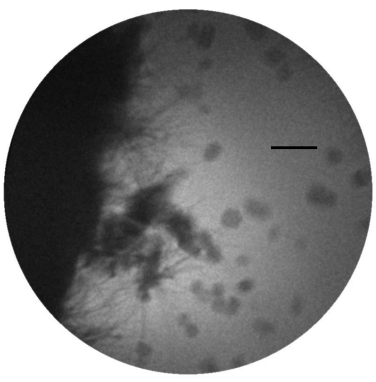

(a) $35 \mathrm{~min}$

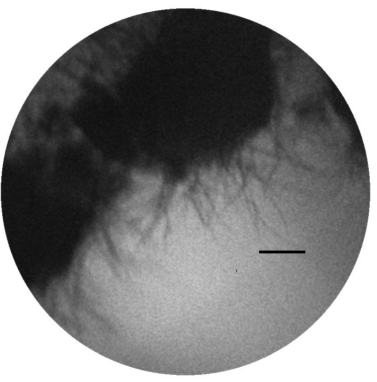

(b) $35 \mathrm{~min}$

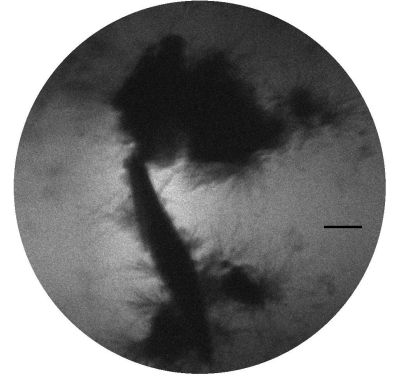

(c) $21 \mathrm{~min}$

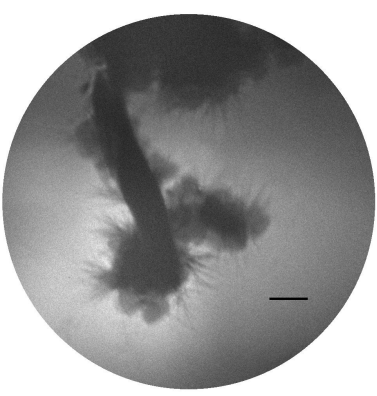

(d) $1 \mathrm{~h} 1 \mathrm{~min}$

Figure 4 - (a) In situ soft x-ray images of around 35 minutes hydration of type I-II cement, (b) commercial expansive admixture hydrated for 35 minutes, (c) and d) blend of type I-II cement and 6\% of expansive admixture over the time in a saturated calcium hydroxide-calcium sulfate solution. Hydration time is indicated. Scale bar corresponds to $1 \mu \mathrm{m}$

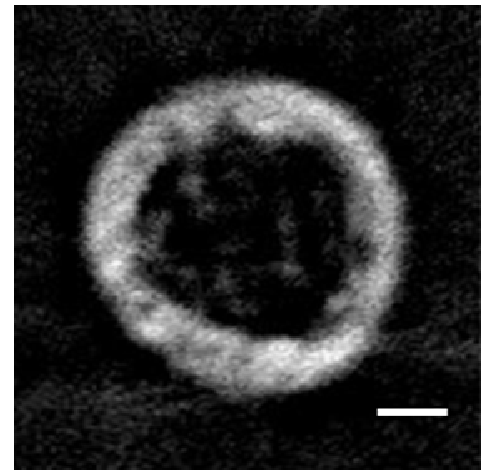

(a) First freeze

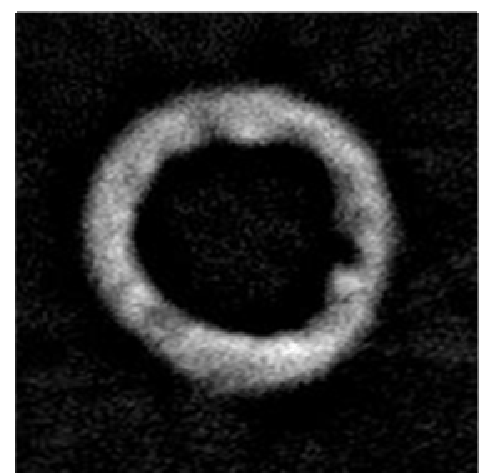

(b) First thaw

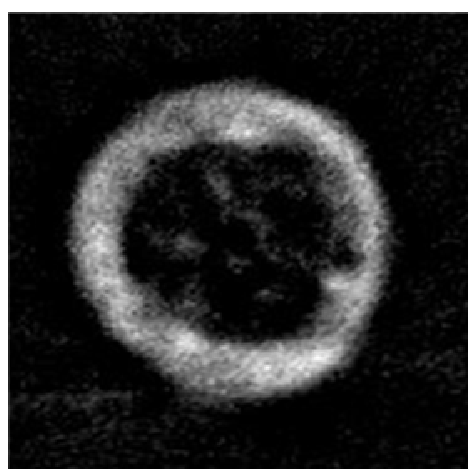

(c) Second Freeze

Figure 5 - Reconstructed tomograms of the sample in the midsection of one of the larger air voids. The scale bar is $50 \mu \mathrm{m}$. The ice crystals (a) in dendritic form; (b) disappear upon thawing; and (c) reappear in changed morphology during the second freeze. 


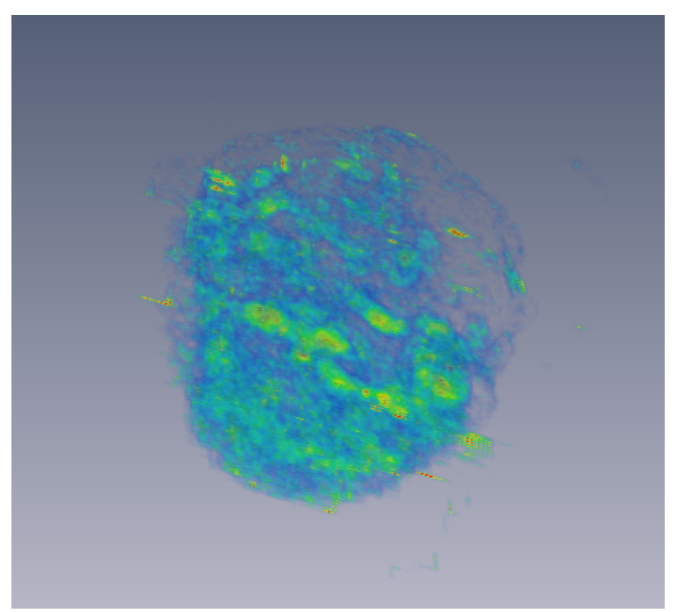

Figure 6 - A 3-D reconstruction of ice formation in a single air void. The cement matrix and the glass capillary have been removed via post reconstruction image processing. The air void is approximately 150 $\mu \mathrm{m}$ in diameter.

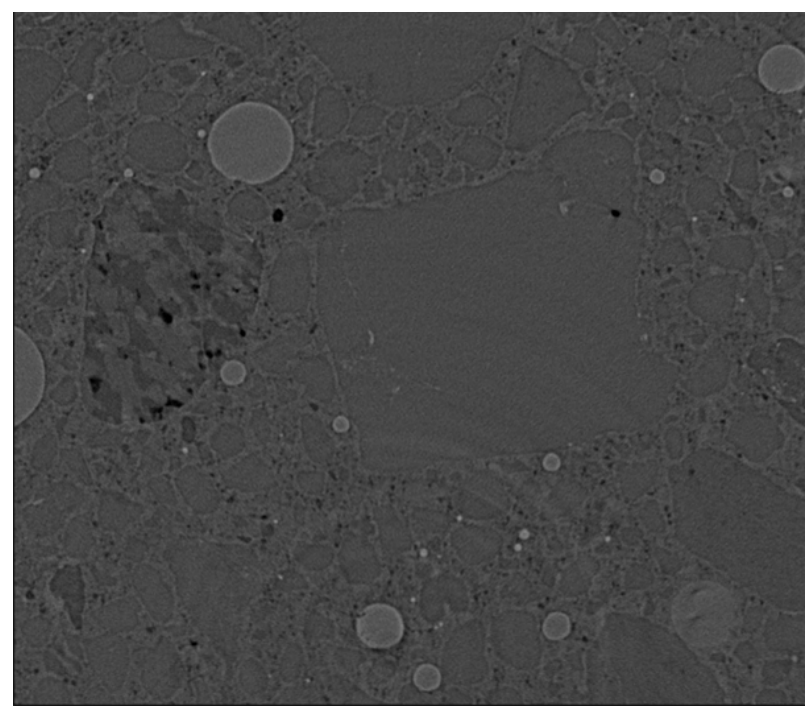

(a) 7 Days

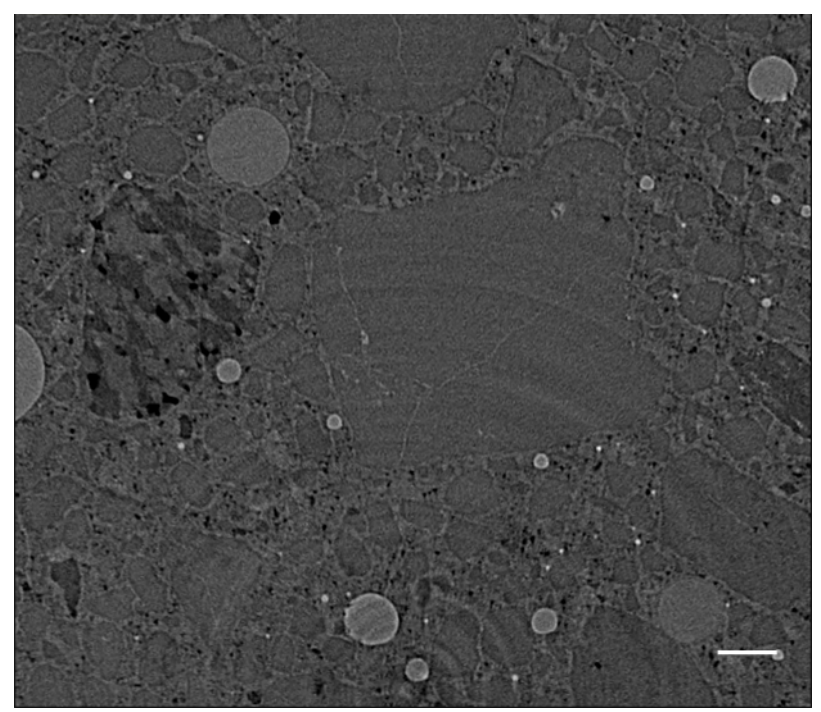

(b) 14 days

Figure 7 - Tomogram of reduced sized mortar bars at (a) 7 days and (b) 14 days of ASTM C1260 testing: New microcrack developments (arrows) are visible. The ring-like structure overlaid on the images is an artifact of the tomographic reconstruction process. The scale bar is $500 \mu \mathrm{m}$. 


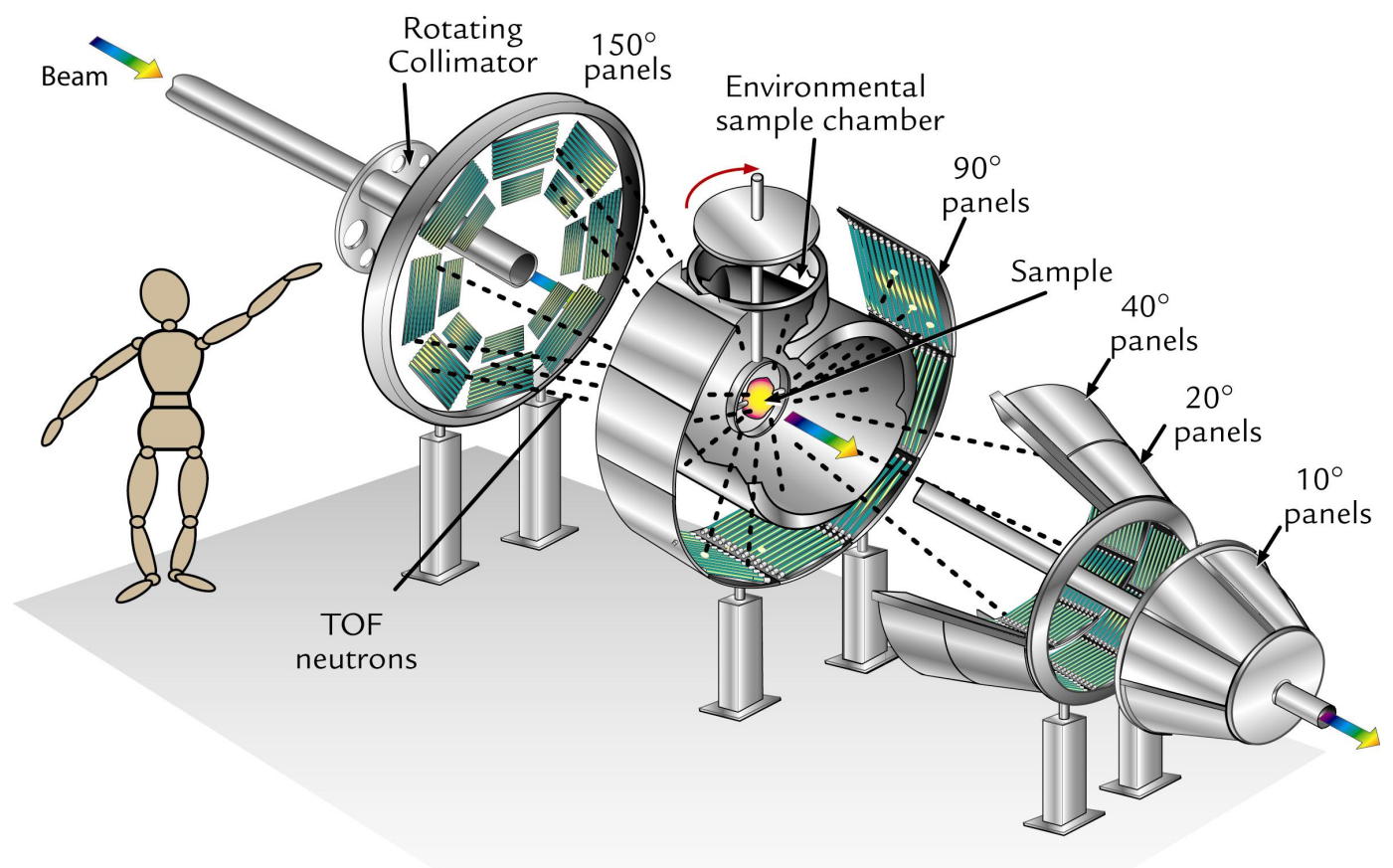

Figure 8 - HIPPO time-of-fight neutron diffractometer at LANSCE with a large sample chamber (person is for scale) and 32 detector panels (green) arranged on 4 banks. Each detector views differently oriented crystals.
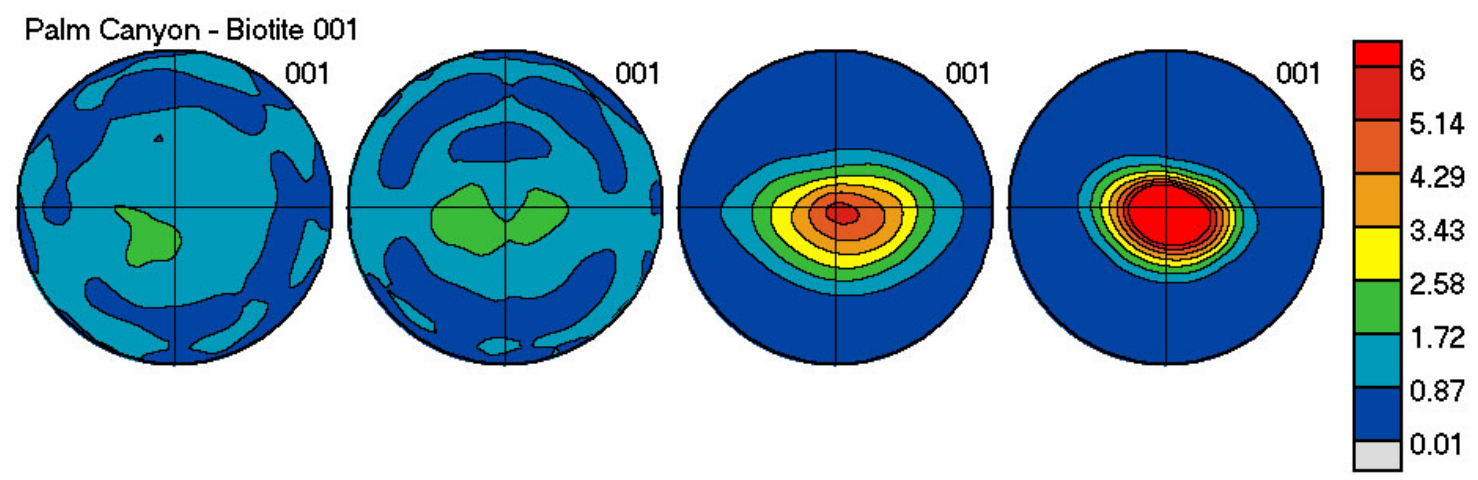

Figure 9 - Pole figures of (001) biotite for selected samples of deformed granite calculated from the orientation distribution based on neutron diffraction data, in order of increasing deformation. The pole figures are projected onto the foliation plane. Linear scale (in m.r.d.), equal area projection. Deformation increases from left to right. 


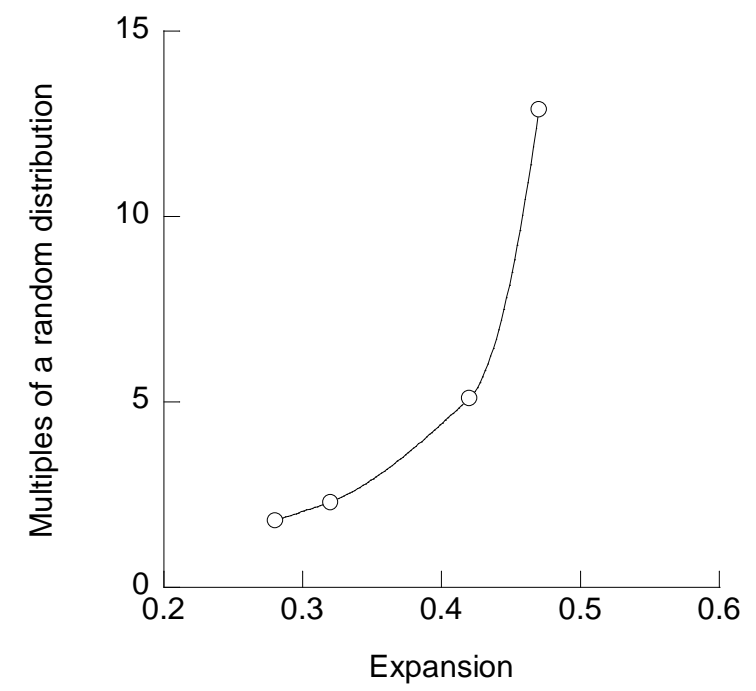

Figure 10 - A plot of the average 30-day expansion value of each rock type from ASTM C 1260 tests versus the (001) pole figure maximum for biotite in multiples of a random distribution. 